\title{
M\&Ms: A software for building realistic Microbial Mock communitieS
}

\author{
Natalia García-García ${ }^{1, *}$, Javier Tamames ${ }^{1}$ and Fernando Puente-Sánchez ${ }^{1, \dagger}$
}

\begin{abstract}
${ }^{1}$ Department of Systems Biology, Address Centro Nacional de Biotecnología (CNB-CSIC), C/ Darwin n 3, Campus de Cantoblanco, 28049, Madrid, Spain.
\end{abstract}

5 †Present address: Department of Aquatic Sciences and Assessment, Swedish University for Agricultural Sciences (SLU), Lennart Hjelms väg 9, 756 51, Uppsala, Sweden

*To whom correspondence should be addressed.

\section{ABSTRACT}

Motivation: Advances in sequencing technologies have triggered the development of many bioinformatic tools aimed to

10 analyze these data. As these tools need to be tested, it is important to simulate datasets that resemble realistic conditions. Although there is a large amount of software dedicated to produce reads from 'in silico' microbial communities, often the simulated data diverge widely from real situations.

Results: Here, we introduce M\&Ms, a user-friendly open-source bioinformatic tool to produce realistic amplicon datasets from reference sequences, based on pragmatic ecological parameters. This tool creates sequence libraries for 'in silico'

15 microbial communities with user-controlled richness, evenness, microdiversity, and source environment. M\&Ms allows the user to generate simple to complex read datasets based on real parameters that can be used in developing bioinformatic software or in benchmarking current tools. M\&Ms also provides additional figures and files with extensive details on how each synthetic community is composed, so that users can make informed choices when designing their benchmarking pipelines.

20 Availability: The source code of M\&Ms is freely available from https://github.com/ggnatalia/MMs

Contact: ngarcia@cnb.csic.es

\section{Introduction}

Microorganisms dwell every habitable environment in the planet's biosphere where they play essential roles in sustaining life. Microbiome studies have sharply increased in the last decades, due in part to a parallel development of sequencing technologies. First studies were performed in extreme environments such as acid mine drainages, where the microbial diversity was low; since then, more complex environments such as gut, marine or soils have been studied (White RA et al., 2016). These microbial communities have different levels of complexity, containing from hundreds to thousands of distinct taxa. Species have been traditionally considered the most significant units in microbial ecology (Shappiro \& Polz, 2014) but, lately, individuals classified within the same species have been involved in different ecological tradeoffs or in different niches (Rasko et al., 2008, Eren et al., 2013, Kashtan et al., 2014, García-García et al., 2019). Thus, in the last decade, the way of study microbial communities has evolved and factors such as microdiversity, referred to the structural and functional diversity below the species level (Schloter et al., 2000), are increasingly relevant.

The most significant technologies for inspecting the profile and function of microbial communities are based on DNA sequencing, like metagenomics and metatranscriptomics. To analyze such amount of data, sophisticated bioinformatic tools have emerged. These new computational methods must be evaluated to ensure a proper functioning. This allows developers to benchmark and compare their software (Baxter et al., 2006, Mangul et al., 2019, Weber et al., 2019). One way to assess the performance of the different algorithms is using simulated data generated on the computer (Engle $e t$ al., 1993, Alosaimi et al., 2020), because often appropriate, real data, are not available. However, choosing an artificial dataset that guarantees an optimal performance is not trivial: the simulated data can be biased by the algorithms, cannot capture true experimental variability or can be less complex than real data (Mangul et al., 2019).

There is a large amount of sequencing simulators such as MetaSIM (Richter et al., 2008), Grinder (Angly et al., 2012), insilicoSeqs (Gourlé et al., 2019), PBSIM2 (Ono et al., 2020), SimuSCoP (Yu et al., 2020) (for a thorough 
review see ref. Alosaimi et al., 2020). These tools are specially designed for simulating read-level artifacts related with sequencing runs or read quality. Their aim is to mimic DNA sequences according to the different sequencing platforms, but not all of them are prepared to simulate realistic microbial communities, or to emulate microbial population heterogeneity.

Other tools are prepared to simulate the dynamics of realistic microbial communities, but they are focused on providing a synthetic 16S rDNA-seq count table that resembles the structure of a microbial community under fluctuating environmental conditions. These tools do not produce simulated sequencing data. Some examples are: the Community Simulator (Marsland et al., 2020) whose aim is simulating microbial population dynamics including environmental conditions throughout time; metaSPARSIM (Patuzzi et al., 2019), a software to generate count matrices resembling real 16S rDNA-seq data; or SPIEC-EASI (Kurz et al., 2015) a sophisticated synthetic microbiome data generator with controllable underlying species interaction topology.

To our knowledge, the first tool designed to produce simulated data from realistic microbial communities is CAMISIM (Fritz et al. 2019). CAMISIM produces automatically metagenomic samples emulating different microbial abundance profiles, multi-sample time series, and differential abundance studies (Fritz et al. 2019). It also includes real and simulated strain-level diversity, and generates sequencing data using taxonomic profiles, or completeley de novo (Fritz et al. 2019). However, CAMISIM does not allow to control community features such as diversity or correlations between taxa.

Selecting the most appropriate bioinformatic tool can be challenging (Mangul et al., 2019) and depends to a large extent on the objectives. The performance tests can contribute the most to the development process and eventually consolidate the tool to handle pragmatic situations (Baxter et al., 2006, Weber et al., 2019). One alternative is the use of the gold standard datasets, usually composed of most known taxa in well characterized environments, but these can hinder the genuine performance of the different tools and lead to overfitting (Mangul et al., 2019).

Here, we describe M\&Ms, a user-friendly tool originally written to generate from simple to complex simulated $16 \mathrm{~S}$ rDNA reads from metagenomic datasets based on desired microbial community's characteristics such as the microdiversity.

\section{Methods}

M\&Ms models a multi-sample microbial abundance profile, includes simulated microdiversity, and generates sequencing data from taxonomic profiles or without them using InSilicoSeqs (Gourlé et al., 2019). M\&Ms aims to produce ecologically meaningful artificial microbial communities by means of an abundance profile based on the evenness and richness of real microbial communities, as well as its microdiversity. It also allows for most frequent taxa per environment thanks to a previous study (Tamames et al., 2016). The software produces a FASTQ file and an abundance profile per sample. Also, it produces a FASTQ file that collects the reads of all the samples and a a mothurformatted groups file with the name of the reads and the sample they belong to.

Simulation with M\&Ms has four stages (Figure 1):

1. Selection of the community members

2. Microdiversity simulation

3. Microbial abundance distribution assignment

4. Sequencing data simulation using InSilicoSeqs (Gourlé et al., 2019) to produce realistic Illumina reads. 

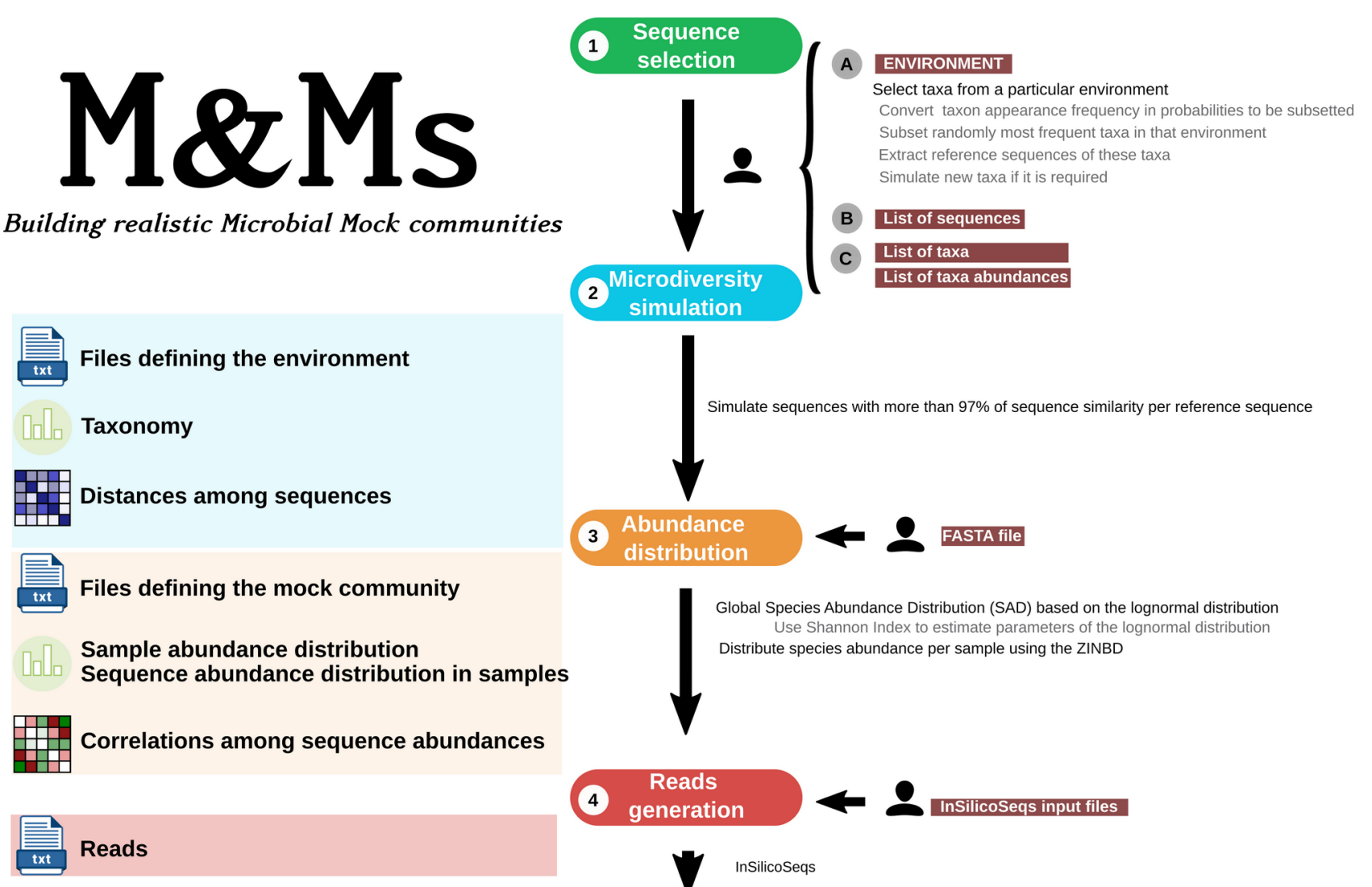

Global Species Abundance Distribution (SAD) based on the lognormal distribution

Use Shannon Index to estimate parameters of the lognormal distribution

Distribute species abundance per sample using the ZINBD

\section{MICROBIAL MOCK COMMUNITY}

Figure 1: M\&Ms pipeline

The software accepts one of the following five independent types of inputs:

- A list of sequences from a particular DB e.g. SILVA database (Yilmaz et al., 2014) from which M\&Ms generates a FASTA file, an abundance profile and the simulated sequencing data.

- A list of taxa from the reference database (and optionally their abundances).

- A pre-existing FASTA file with the desired sequences, on the basis of which M\&Ms produces the simulated abundance profile and the data.

- A list of 16S rRNA gene sequences and their abundances, so that only simulated sequencing data will be generated.

- A particular environment, so most of the taxa will be selected from that environment, according to a reference table with most frequent taxa (at the genus level) per environment. M\&Ms will produce a FASTA file with sequences from species from that environment, an abundance profile and the simulated sequencing data.

To select taxa from a particular environment, M\&Ms uses a genus per environment matrix derived in a previous work (Tamames et al., 2016)(see Supplementary Note 1 for details). For a given pool of possible reference sequences, M\&Ms simulates its corresponding microdiversity introducing point mutations.

We use a log-normal distribution to predict the global species abundance distribution (SAD) of the artificial community. Although, since the 1930s, ecologists have developed different models to predict the SAD (McGuill, 2010), those based on the log-normal are considered to be standards to test new models (Shoemaker et al, 2017). The lognormal is characterized by a right-skewed frequency distribution that becomes approximately normal under logtransformation. This model reflects the 'rare biosphere' (i.e. the fact that most species in a given sample will be present 
bioRxiv preprint doi: https://doi.org/10.1101/2021.04.21.440404; this version posted April 22, 2021. The copyright holder for this preprint (which was not certified by peer review) is the author/funder, who has granted bioRxiv a license to display the preprint in perpetuity. It is made available under aCC-BY-NC 4.0 International license.

in very low abundances), which is one of the most intensively studied patterns of microbial diversity (Pedrós Alió, 2012; Shoemaker et al., 2017).

The probability density function of the log normal distribution is defined by the mean $\mu$ and standard deviation $\sigma$, which affects the shape of the distribution. Longuet-Higgins and also Edden (Longuet-Higgins, 1971; Edden, 1971) studied the relation between species diversity and the log-normal distribution of individuals among species. Both proposed the use of the logarithmic standard deviation, $\sigma$, as an indicator of the unevenness of species distribution. In particular, $\sigma$ and the number of species correlates well with the values given by the Shannon and Weaver diversity index (Longuet-Higgins 1971; Edden 1971). The Shannon index (H) is an index commonly used to characterize species diversity in a community that accounts for both abundance and evenness of the species present (Shannon 1948, Hill et al., 2002). We calculate the standard deviation of the log-normal distribution from a specific Shannon Index to approximate the SAD of the mock community, thus the species abundance distribution is more realistic and allow M\&Ms to design ecologically meaningful mock communities unlike other equivalent tools. A more detailed explanation of this approximation between Shannon diversity index and the standard deviation of a log-normal distribution can be found in Supplementary Note 2.

We then determine the abundance distribution of the different simulated individuals using the Zero-Inflated Negative Binomial Distribution (ZINBD). We use the NORmal-To-Anything (NORTA) approach, which is an approximate technique to generate arbitrary continuous and discrete multivariate distributions from a target correlation matrix, using a multivariate normal (Yahav \& Shmuelli, 2011; Kurz et al., 2015). First, we sample from a multivariate normal distribution with zero mean and standard deviation one using a randomly created correlation matrix. For each probability, the Normal cumulative distribution function (CDF) is transformed to the target distribution, in this case, the ZINBD via its inverse CDF (Yahav \& Shmuelli, 2011; Kurz et al., 2015). The target distribution is the ZINBD which has been observed to fit properly microbiome data, characterized by having an increasing number of zeros at lower taxonomic levels (Xu et al., 2015; Xia \& Sun, 2017).

Finally, M\&Ms runs InSilicoSeqs (Gourlé et al., 2019) to simulate metagenomic Illumina sequencing samples with the designed abundance profiles.

\section{Results}

M\&Ms has been designed to produce artificial communities, which can be used to test new software in different stages of development and benchmarking. We envisage three different situations: simple mock communities with few sequences (useful in initial stages of software development), complex artificial communities but just one or more samples, convenient in intermediate steps of software development. Additionally, it also facilitates the option of working with the same mock community but with different simulated sequencing parameters in a straightforward way.

M\&Ms provides also plots and extra files to effortlessly visualize the main characteristics of the mock community. With all this information, the user can handily establish comparisons among the initial composition of the community and the results of applying any tool. Also, M\&Ms have been develop to be flexible, so adding extra improvements such as a better algorithm to simulate microdiversity can be effortlessly implemented.

Therefore, the main advantage of M\&Ms consists of automatically obtaining realistic microbial mocks with ease. A comparison with similar tools that apart from generating DNA data, are able to simulate microorganisms distribution is displayed in Supplementary Note 3. 
bioRxiv preprint doi: https://doi.org/10.1101/2021.04.21.440404; this version posted April 22,2021 . The copyright holder for this preprint (which was not certified by peer review) is the author/funder, who has granted bioRxiv a license to display the preprint in perpetuity. It is made available under aCC-BY-NC 4.0 International license.

\section{Funding}

Computational resources were provided by the Spanish Ministry of Economy of Competitiveness grants CTM201680095-C2-1-R and PID2019-110011RB-C31 from the Spanish Ministerio de Economía, Industria y Competitividad. NG-G was funded by a grant from the Severo Ochoa Program at CNB (SEV-2013-0347-17-2). FP-P was funded by a grant fron Juan de la Cierva (IJC2018-035180-I), both grants from the Spanish Ministry of Science and Innovation. FPP was also funded by Marie Skłodowska-Curie IF Action 892961 - ARAMIS from the European Research Council.

Conflict of Interest: The authors declare that they have no competing interests.

\section{References}

- $\quad$ Alosaimi S, Bandiang A, van Biljon N, Awany D, Thami PK, Tchamga MSS, Kiran A, Messaoud O, Hassan RIM, Mugo J, Ahmed A, Bope CD, Allali I, Mazandu GK, Mulder NJ, Chimusa ER. (2020). A broad survey of DNA sequence data simulation tools. Briefings in Functional Genomics; 19(1):49-59.

- Angly FE, Willner D, Rohwer F, Hugenholtz P, \& Tyson GW. (2012). Grinder: a versatile amplicon and shotgun sequence simulator. Nucleic acids research; 40(12): e94.

- Baxter SM, Day SW, Fetrow JS, Reisinger SJ (2006) Scientific Software Development Is Not an Oxymoron. PLoS Computational Biology; 2(9): e87.

- $\quad$ Edden AC. (1971). A measure of species diversity related to the lognormal distribution of individuals among species. Journal of Experimental Marine Biology and Ecology; 6: 199-209.

- $\quad$ Engle ML, Burks C. (1993). Artificially generated data sets for testing DNA sequence assembly algorithms. Genomics; 16(1):286-8.

- Eren AM, Maignien L, Sul WJ, Murphy LG, Grim SL, Morrison HG, Sogin ML. (2013) Oligotyping: Differentiating between closely related microbial taxa using 16S rRNA gene data. Methods in Ecology and Evolution; 4(12):1111-1119.

- $\quad$ Fritz A, Hofmann P, Majda S, Dahms E, Dröge J, Fiedler J, Lesker TR, Belmann P, DeMaere MZ, Darling AE, Sczyrba A, Bremges A \& McHardy AC. (2019). CAMISIM: simulating metagenomes and microbial communities. Microbiome; 7(1): 17.

- García-García, N, Tamames, J, Linz, AM, Pedrós-Alió, C, \& Puente-Sánchez, F. (2019). Microdiversity ensures the maintenance of functional microbial communities under changing environmental conditions. The ISME journal; 13(12): 2969-2983.

- Gourlé H, Karlsson-Lindsjö O, Hayer J \& Bongcam-Rudloff E. (2019). Simulating Illumina metagenomic data with InSilicoSeq. Bioinformatics; 35(3): 521-522.

- Hill TC, Walsh KA, Harris JA, Moffett BF. (2003). Using ecological diversity measures with bacterial communities. FEMS Microbiology Ecology; 43(1): 1-11.

- Kashtan N, Roggensack SE, Rodrigue S, Thompson JW, Biller SJ, Coe A, Ding H, Marttinen P, Malmstrom RR, Stocker R, Follows MJ, Stepanauskas R, Chisholm SW. (2014) Single-cell genomics reveals hundreds of coexisting subpopulations in wild Prochlorococcus. Science; 344(6182): 416-420.

- $\quad$ Kurtz ZD, Müller CL , Miraldi ER, Littman DR, Blaser MJ, Bonneau RA. (2015). Sparse and Compositionally Robust Inference of Microbial Ecological Networks. PLOS Computational Biology; 11(5): e1004226.

- Longuet-Higgins MS. (1971). On the Shannon-Weaver index of diversity, in relation to the distribution of species in bird censuses. Theoretical Population Biology; 2(3): 271-289.

- Mangul S, Martin LS, Hill BL, Lam AK, Distler MG, Zelikovsky A, Eskin E \& Flint J. (2019). Systematic benchmarking of omics computational tools. Nature communications; 10(1): 1393.

- Marsland R, Cui W, Goldford J \& Mehta P. (2020). The Community Simulator: A Python package for microbial ecology. PloS one; 15(3): e0230430.

- $\quad$ McGill BJ. (2010). Towards a unification of unified theories of biodiversity. Ecology Letters; 13(5): 627-642.

- Ono Y, Asai K, Hamada M. (2013). PBSIM: PacBio reads simulator--toward accurate genome assembly. Bioinformatics; 29(1):119-121. 
bioRxiv preprint doi: https://doi org/10.1101/2021.04.21.440404; this version posted April 22, 2021. The copyright holder for this preprint (which was not certified by peer review) is the author/funder, who has granted bioRxiv a license to display the preprint in perpetuity. It is made available under aCC-BY-NC 4.0 International license.

- Patuzzi I, Baruzzo G, Losasso C, Ricci A \& Di Camillo B. (2019). metaSPARSim: a 16S rRNA gene sequencing count data simulator. BMC bioinformatics; 20(9), 416.

- $\quad$ Pedrós-Alió C. (2012). The rare bacterial biosphere. Annual Review of Marine Science; 4: 449-66.

- $\quad$ Rasko DA, Rosovitz MJ, Myers GS, Mongodin EF, Fricke WF, Gajer P, Crabtree J, Sebaihia M, Thomson NR, Chaudhuri R, Henderson IR, Sperandio V, \& Ravel J. (2008). The pangenome structure of Escherichia coli: comparative genomic analysis of E. coli commensal and pathogenic isolates. Journal of bacteriology; 190(20): 6881-6893.

- $\quad$ Richter DC, Ott F, Auch AF, Schmid R, \& Huson DH. (2008). MetaSim: a sequencing simulator for genomics and metagenomics. PloS one; 3(10): e3373.

- $\quad$ Schloter M, Lebuhn M, Heulin T, Hartmann A. (2000). Ecology and evolution of bacterial microdiversity. FEMS Microbiol Reviews; 24(5):647-60.

- Shannon, CE. (1948), A Mathematical Theory of Communication. Bell System Technical Journal, 27: 379423.

- $\quad$ Shapiro BJ \& Polz MF. (2014). Ordering microbial diversity into ecologically and genetically cohesive units. Trends in Microbiology; 22(5):235-247

- Shoemaker WR, Locey KJ, Lennon JT. (2017). A macroecological theory of microbial biodiversity. Nature Ecology and Evolution; 1(5):107.

- Tamames J, Sánchez PD, Nikel PI, \& Pedrós-Alió C. (2016). Quantifying the Relative Importance of Phylogeny and Environmental Preferences As Drivers of Gene Content in Prokaryotic Microorganisms. Frontiers in microbiology; 7, 433.

205 - Weber LM, Saelens W, Cannoodt R, Soneson C, Hapfelmeier A, Gardner PP, Boulesteix AL, Saeys Y, Robinson MD. (2019). Essential guidelines for computational method benchmarking. Genome Biology; 20(1): 125.

- White RA, Callister S, Moore R, Baker E \& Jansson JK. (2016). The past, present and future of microbiome analyses. Nature Protocols; 11: 2049-2053.

$210 \quad$ - Xia Y, Sun J. (2017). Hypothesis Testing and Statistical Analysis of Microbiome. Genes \& Diseases; 4(3): 138-148.

- Xu, L, Paterson AD, Turpin W, \& Xu W. (2015). Assessment and Selection of Competing Models for ZeroInflated Microbiome Data. PloS one; 10(7): e0129606.

- Yahav I \& Shmueli G. (2012). On generating multivariate Poisson data in management science applications. Applied Stochastic Models in Business and Industry; 28: 91-102.

- Yilmaz P, Parfrey LW, Yarza P, Gerken J, Pruesse E, Quast C, Schweer T, Peplies J, Ludwig W \& Glöckner FO. (2014). The SILVA and "All-species Living Tree Project (LTP)" taxonomic frameworks. Nucleic acids research; 42(Database issue), D643-D648.

- Yu Z, Du F, Ban R, \& Zhang Y. (2020). SimuSCoP: reliably simulate Illumina sequencing data based on position and context dependent profiles. BMC bioinformatics; 21(1), 331. 